\title{
Osteonecrosis of the jaw in the era of targeted therapy and immunotherapy in oncology
}

\author{
Antonio Fabrizio Nifosi ${ }^{1}$, Mariateresa Zuccarello ${ }^{2}$, Lorenzo Nifosi ${ }^{3}$, Vanessa Hervas Saus ${ }^{4}$, Gianfilippo Nifosi ${ }^{5}$ \\ ${ }^{1}$ Dental Surgeon, Forli, ${ }^{2}$ Department of Medicine, University of Catania, Catania, Italy, \\ Departments of ${ }^{3}$ Dentistry and ${ }^{4}$ Dental Hygiene, European University of Valencia, Valencia, Spain, \\ ${ }^{5}$ Hemato-Oncology Clinic, Department of Internal Medicine, Brugmann University Hospital Center, Brussels, Belgium
}

\begin{abstract}
J Korean Assoc Oral Maxillofac Surg 2019;45:3-8)
Osteonecrosis of the jaw (ONJ) is a well-known pathological condition in oncology derived from the use of bisphosphonates (BPs) and denosumab. Many molecular and immunological targets have been introduced for daily use in cancer treatment in recent years; consequently, new cases of ONJ have been reported in association with these drugs, especially if administered with BPs and denosumab. When the drugs are administered alone, ONJ is rarely seen. The objective of our study was to analyze the recent literature relative to the association of ONJ with these new drugs highlighting the pathogenic, clinical and therapeutic aspects. The close collaboration between maxillofacial surgeon, oncologist, dentist, and dental hygienist remains the most important aspect for the prevention, prompt recognition, and treatment of this pathology.
\end{abstract}

Key words: Bone, Infection, Oral manifestations, Immunomodulation, Angiogenesis modulators

[paper submitted 2018. 3. 11 / revised 2018. 5. 24 / accepted 2018. 5. 27]

\section{Introduction}

Medication-related osteonecrosis of the jaw (MRONJ) is a condition of exposed bone or bone that can be seen through an intraoral or extra-oral fistula in the maxillofacial region, which occurs for more than 8 weeks in patients receiving drugs without a history of head and neck radiation therapy. This definition includes cases of osteonecrosis of the jaw (ONJ) associated with antiresorptive drugs, such as bisphosphonates (BPs) and denosumab. In recent years, the number of MRONJ cases has been constantly increasing due to new molecular target and immunological drugs introduced for cancer treatment. Most of these new drugs have mechanisms of action involving the targets listed in Table 1 that are poten-

\section{Gianfilippo Nifosì}

Hemato-Oncology Clinic, Department of Internal Medicine, Brugmann University Hospital Center, 4 Van Gehuchten Square, 1082 Brussels, Belgium

TEL: +32-2-477-31-65 FAX: +32-477-35-29

E-mail: g.nifosi@tin.it

ORCID: https://orcid.org/0000-0003-4329-6297

(c) This is an open-access article distributed under the terms of the Creative Commons Attribution Non-Commercial License (http://creativecommons.org/ licenses/by-nc/4.0/), which permits unrestricted non-commercial use, distribution, and reproduction in any medium, provided the original work is properly cited. Copyright (C) 2019 The Korean Association of Oral and Maxillofacial Surgeons. All rights reserved. tially responsible for MRONJ. MRONJ is probably caused by multiple factors with the participation of genetic polymorphisms and drug interactions. Duration of drug use, concomitant treatments, old age, and other pathologies are important predisposing systemic factors for MRONJ. Tooth extraction, local anatomic factors, and concomitant oral diseases are also important triggering factors ${ }^{1}$. The purpose of this review article was to summarize the medicaments associated with MRONJ that were added in the American Association of Oral and Maxillofacial Surgeons (AAOMS) position paper in 2014.

\section{Tyrosine Kinase Inhibitors (TKIs)}

Sorafenib is approved for renal and liver cancer treatment. This drug inhibits several tyrosine kinases, including vascular endothelial growth factor receptor (VEGFR), plateletderived growth factor receptor (PDGFR), and Raf kinases ${ }^{2}$. The conversion of normal cells to tumor cells is guided by molecular pathways, including the Raf/Mek/Erk pathway, which sorafenib has the capacity to inhibit. Sunitinib, like sorafenib, inhibits the kinase pathways of tumor cells and angiogenesis ${ }^{3}$. This drug is metabolized by the liver, with a halflife of 40 to 60 hours. An important collateral effect is the 
Table 1. Potential mechanisms of medication-related osteonecrosis of the jaw

\begin{tabular}{ll} 
Suppression of bone turnover & Compromised bone microenvironment functioning affecting bone remodeling and repair \\
Angiogenesis inhibition & Antiangiogenic affects delaying wound healing and/or affecting micro-infarction in bone and/or soft tissues \\
Infection and inflammation & Microorganisms of the oral cavity promoting cell death in the bone and/or soft tissues \\
Soft tissue toxicity & Direct toxicity toward oral epithelial cells \\
Immune-related & Innate/acquired immune system involvement \\
\hline
\end{tabular}

Antonio Fabrizio Nifosi et al: Osteonecrosis of the jaw in the era of targeted therapy and immunotherapy in oncology. J Korean Assoc Oral Maxillofac Surg 2019

appearance of ONJ, specifically if the drug is associated with intravenous BPs. The first description of ONJ based on TKI treatment dates back to $2009^{4}$. In patients with advanced renal cancer, there is a $10 \%$ risk of ONJ with concomitant antiVEGFR-TKI and BP therapy. It is possible that this incidence is underestimated because most patients are within a short survival percentage. In fact, in patients with $>12$ months of BP administration showed a risk of $>17 \%{ }^{5}$. Several case reports described high risk association with zoledronic acid and sunitinib $^{6-8}$. Recently, a global MRONJ-incidence of $11 \%$ in patients receiving concomitant treatment with VEGFR-TKIs and bone resorption inhibitors has been reported ${ }^{9}$ and the risk is very high if BPs were previously administered. The risk of transformation of induced chemotherapy oral mucositis to bisphosphate-related ONJ (BRONJ) has been described. Several case reports have demonstrated an association between sunitinib alone and ONJ, after one-year treatment ${ }^{10,11}$. The first case of ONJ in a cirrhotic patient with hepatocellular carcinoma treated with sorafenib has been described ${ }^{12}$. Periodontal and dental infections, and dental extractions are the main risk factors ${ }^{13}$.

Imatinib mesylate is a chemotherapeutic drug that inhibits many tyrosine kinase enzymes involved in an important signal transduction pathway that leads to DNA duplication and/ or gene transcription. Imatinib is specific for the $T K$ domain of the protein encoded by Abelson proto-oncogene ( $a b l)$, and inhibits c-Kit and $P D G F-R^{14}$. It is currently used to treat chronic myeloid leukemia (CML), acute lymphoblastic leukemia (ALL), and gastrointestinal stromal tumors (GISTs). Some cases of ONJ have been reported after 22 months of imatinib monotherapy. Some evidence suggests that this side effect may be attributed to the inhibition of c-Kit and c-Fms signaling, which is carried out directly by imatinib. This inhibition leads to a decrease in osteoclast activity and global dysregulation of bone remodeling. Imatinib interferes with the protective activity produced by carbonic anhydrase II on bone and also inhibits PDGFR, which reduces the activity of osteoclastogenic cytokines including macrophage colonystimulating factor (M-CSF) and receptor activator of nuclear factor kappa-B ligand (RANKL). Lastly, imatinib inhibits os- teoblastic cell proliferation ${ }^{15}$. Even in this case, the osteonecrosis that occurs can be considered as MRONJ ${ }^{16}$. Recently, cases associated with dasatinib, another TKI used in the treatment of CML and acute BCR/ABL ALL and erlotinib, a TKI used in the treatment of non-small cell lung cancer, have been reported $^{17}$.

\section{Mammalian Target of Rapamycin Inhibitors (mTOR Inhibitors)}

The mammalian target of the rapamycin pathway (mTOR) plays a key role in regulating bone homeostasis. mTOR signaling regulates various bone cell populations, which results in changes of bone homeostasis ${ }^{18}$. Everolimus is a 40-O-(2hydroxyethyl) derivative of sirolimus and similarly inhibits activity of the mTOR pathway. It is currently used as an immunosuppressant to prevent transplant rejection and is likewise used in the treatment of neuroendocrine tumors of pancreatic origin ( $\mathrm{pNET}$ ), renal cell carcinoma, and breast cancer. Temsirolimus is a specific inhibitor of mTOR that, even though it shows activity on its own, is also known to be converted to sirolimus (rapamycin) in vivo. Temsirolimus is indicated for high-risk, advanced renal cell carcinoma and relapsed or refractory mantle cell lymphoma treatment.

mTOR drugs bind to FKBP-12, an intracellular protein, which causes a lower expression of D-type cyclins, c-Myc, and ornithine decarboxylase, regulatory proteins of the cell cycle that result in G1 phase cell cycle arrest. However, mTOR inhibition reduces hypoxia-inducible factors (HIF-1 and HIF-2 $\alpha$ ) and vascular endothelial growth factor (VEGF) production, which blocks tumor angiogenesis ${ }^{19}$. Moreover, the mTOR pathway is essential for both the innate and adaptive immune responses and its inhibition causes cell growth arrest and often immunosuppression. The immunosuppressive state explains the infection susceptibility of the treated patient. In addition, preclinical studies in mouse models have demonstrated that genetic and pharmacological inhibition of mTOR inhibited osteoclastogenesis and increased apoptosis of osteoclasts ${ }^{20}$. Furthermore, in breast cancer, mTOR inhibition in tumor cells by everolimus leads to a suppres- 
sion of paracrine pro-osteoclast activity by interfering with the nuclear factor kappa-light-chain-enhancer of activated B cells $(\mathrm{NF \kappa B})$ pathway. As a result, this may also account for the delayed progression of bone metastatic disease ${ }^{21}$ and could explain the mechanism of ONJ appearance associated with mTOR inhibitors. The first case of ONJ was reported in a patient with thyroid cancer treated with a $10-\mathrm{mg}$ daily dose of everolimus for three years ${ }^{22}$. The second ONJ case was a breast cancer patient treated with a 20-mg daily dose of everolimus and a 25-mg daily dose of examestane ${ }^{23}$. Giancola et al. ${ }^{24}$ reported a patient treated with everolimus for lung metastasis with ONJ appearing the next year. Recently, we described a case of ONJ related to temsirolimus and denosumab treatment, in which temsirolimus can play a co-factor role in the development and maintenance of denosumab-related ONJ (DRONJ) due to its antiproliferative, antiangiogenic, and immunosuppressive effects ${ }^{25}$.

\section{Anti-angiogenics}

VEGF is an endothelial cell-specific mitogen in vitro and an angiogenic inducer in vivo. Its action is mediated by interaction with two high affinity receptors, VEGFR-1 (tyrosine kinase Flt-1) and VEGFR-2 (Flk-1/KDR), on the surface of endothelial cells. Therefore, VEGF plays an essential role in angiogenesis and is important for bone angiogenesis. It is regulated by two sets of molecules with opposing functions: proangiogenic molecules (such as VEGF) and anti-angiogenic molecules (such as thrombospondin-1). Under homeostatic conditions, pro/anti-angiogenic balance is shifted toward anti-angiogenic factors resulting in quiescent blood vessels. On the other hand, in neoplastic lesions, angiogenic balance is shifted towards pro-angiogenesis. Thus, cancer hypoxia is considered to be the primary causal factor for this switch. The release of pro-angiogenic factors from tumor cells and host cells (fibroblasts and macrophages) causes disruption of the surrounding basement membrane vasculature, which is attributed to the activation of a group of proteases including plasminogen activator and collagenases. Furthermore, these pro-angiogenic factors are strong chemotactic factors for endothelial cells and attract circulating bone marrow progenitor cells stimulating their differentiation into endothelial cells. Then, new basement membrane is formed, and pericytes are attracted to surround the neo-vessels. These neo-vessels are characterized by increased permeability and leakiness. Angiogenesis is involved in tumor growth, invasion, and metastasis $^{26}$. The gene encoding VEGF-A was cloned in 1989 and is considered a mediator of tumoral angiogenesis that is overexpressed in various human tumors; therefore, blocking VEGF can have an anticancer role.

Bevacizumab (Avastin) is a recombinant, humanized antiVEGF monoclonal antibody that binds to VEGF-A and blocks the angiogenic process. It is approved for clinical use in metastatic colorectal, non-small cell lung, breast, ovarian, and cervical cancer, and glioblastoma multiforme. Thromboembolic episodes, hypertension, hemorrhage, gastrointestinal perforation, and wound healing complications are common and are potentially serious side effects of bevacizumab. Cases of isolated soft tissue necrosis after dental extraction ${ }^{27}$, laryngeal necrosis ${ }^{28}$, and nasal septum perforation ${ }^{29}$ have been reported. Bevacizumab treatment has to be stopped between 5 and 8 weeks before dental extraction or surgery. Conversely, bevacizumab-related ONJ is rare. The first case occurring in a female patient with a history of breast cancer who was treated with intravenous bevacizumab and oral capecitabine was described by Estilo et al. ${ }^{30}$ in 2008. A second case was described in the same year in a female breast cancer patient who received bevacizumab and liposomal doxorubicin ${ }^{31}$. Subsequently, other cases were reported with ${ }^{32-34}$ or without $t^{35,36}$ association of BPs. A failure to repair physiological trauma (tooth brushing or chewing) and a possible correlation with eruption of the lower third molar tooth in ONJ development following bevacizumab treatment have been hypothesized ${ }^{37}$. There is no reliable epidemiological data on the incidence of bevacizumab-related ONJ. Retrospective studies report that anti-angiogenic agents (bevacizumab and sunitinib) in combination with BPs induce ONJ with greater frequency (16\%), but there is no data on bevacizumab-ONJ incidence ${ }^{38}$. Higher than usual incidence of ONJ (18.3\% without BPs and 20\% with BPs) was reported in a series of patients treated with bevacizumab, thalidomide, docetaxel, and prednisone for metastatic prostate cancer ${ }^{39}$. The authors hypothesized an enhanced effect of known anti-angiogenic drugs (bevacizumab and thalidomide) with chemotherapy and steroids. Guarneri et al. ${ }^{40}$ in 2010 investigated the databases of three clinical trials, AVADO, RIBBON-1, and ATHENA, where bevacizumab was studied in 3,560 patients with locally recurrent or metastatic breast cancer. The overall incidence of bevacizumabrelated ONJ was $0.3 \%$ to $0.4 \%$, considerably lower than previously suggested with anti-angiogenic therapy in a small retrospective analysis. There was a trend towards increased ONJ incidence in patients who received BP-associated therapy $(0.9 \%-2.4 \%)$, that is within the $1 \%$ to $6 \%$ range reported for BPs alone. The authors attributed great importance 
to good oral hygiene, dental examination, and avoidance of invasive dental procedures for $\mathrm{ONJ}$ prevention in this population of patients. These results are consistent with the Memorial Sloan Kettering Cancer Center retrospective analysis of 1,711 patients with cancer, in which the incidence of ONJ was $0 \%$ in bevacizumab treatment without BPs, and $2 \%$ with $\mathrm{BPs}^{41}$. No ONJ cases were reported in 59 bone metastatic cancer patients (34 with breast and 25 with non-small cell lung cancer) treated with bevacizumab and zoledronic acid, when a baseline dental examination and preventive dental measures were adopted ${ }^{42}$. Therefore, bevacizumab does not seem to be an important risk factor in the development of ONJ in this patient group.

Hopp et al. ${ }^{43}$ in 2012 reported the first case of ONJ associated with intravitreal administration of VEGF for two years for retinal vascular thrombosis. At the bone level, the drug, in synergy with M-CSF, induces monocyte and macrophage differentiation and chemotaxis. It also stimulates the function of osteoclasts and is essential for osteogenic differentiation and bone formation, and directly stimulates osteoclastic bone resorption. Finally, VEGF-controlled angiogenesis is also required for bone remodeling and repair. Consequently, inhibiting osteoclasts and neoangiogenesis, bevacizumab compromises micro normal bone remodeling and vessel integrity in the mandible. These effects are reinforced by the regression of existing microvessels, inhibition of vessel growth, and neovascularization. Likewise, it can cause oral mucosal breakdown and can hamper mucosal repair, which may result in necrotic jawbone exposure. Finally, reduction of the host defense mechanisms, secondary to inhibition of monocyte and macrophages chemotaxis and differentiation, can trigger necrotic bone over-infection. Bevacizumab-related ONJ is characterized by early appearance (in 3 months) and disappearance (in 3 months). In fact, the anti-angiogenic and antiresorptive effects of bevacizumab are dose-dependent and time-dependent ${ }^{44}$. Compared to BRONJ, it is less destructive and heals spontaneously with conservative treatment. An important effect in the pathogenesis is the ability of this drug to reduce mucosal healing capacity that could worsen dental and periodontal infection, with rapid propagation to the underlying bone ${ }^{45}$. Finally, the anti-angiogenic effect of bevacizumab is increased by other drugs such as temsirolimus ${ }^{46}$.

Ziv-aflibercept is a recombinant protein composed of human VEGF receptor extracellular domains and the Fc portion of human immunoglobulin G1. This molecule is currently used for the treatment of several solid tumors, including refractory metastatic colorectal cancer. Ziv-aflibercept binds to circulating VEGFs and acts like a "VEGF trap". It blocks vascular growth and vascular permeability in the tumour ${ }^{47}$. The literature reports cases of patients treated with Zivaflibercept that develop MRONJ after 3.5 to 11.5 months. This could be explained by the anti-angiogenic activity of the drug, since it has mechanisms of action that are similar to $\mathrm{BPs}^{48,49}$.

\section{Immunological Drugs}

Ipilimumab is a humanized monoclonal $\mathrm{IgG} 1 / \mathrm{k}$ antibody against CTLA-4 (cytotoxic T-lymphocyte-associated antigen-4) expressed by suppressor T-regulatory cells and activated T-cells. It binds with antigen presenting cells to reduce the T-lymphocyte dependent immune response. Ipilimumab improves the responses of activated T-cells against tumors and increases their number ${ }^{50}$. It has shown benefits in the treatment of advanced melanoma and in other advancedstage malignancies. The literature describes how the systemic activation of T-cells implies the production of OPGL (osteoprotegerin ligand), whose corresponding receptor (RANK) is expressed on mature osteoclast and chondrocytes. The interaction between OPGL and RANK leads to osteoclastogenesis and resultant bone loss. This has led the authors to deduce that activated T-cells could directly influence bone metabo$\operatorname{lism}^{51}$, which could realize a condition of vulnerability of the bone. That, together with mild trauma like tooth brushing, can lead to ONJ and subsequent bone exposure. Currently, few cases of this side effect are reported in literature, probably because treatment with ipilimumab is usually shortterm $^{52}$.

\section{MRONJ Prevention}

The American Dental Association (ADA) has reported that good oral hygiene is the best way to reduce the risk of $\mathrm{ONJ}^{53}$. Tooth brushing twice a day with a fluoride paste is important for good oral hygiene, especially if associated with the use of dental floss and mouthwash. The best amount of daily brushing, flossing, and washing useful for the prevention of ONJ has not been demonstrated. It is important to identify smoking patients, patients with a poor oral hygiene, and systemic pathologies such as diabetes that increase the risk of oral infections ${ }^{54}$. Oral surgeons will have to complete every necessary procedure before oncology therapy, give appropriate antibiotic therapy, and close the wound following tooth extraction. A multidisciplinary approach between the oncolo- 
gist, dentist, maxillofacial surgeon, and dental hygienist is a good strategy for prevention. The role of dental hygienists in the education of these patients is essential. Recommendations include antimicrobial mouth rinsing, tartar removal, and maintenance of good oral hygiene ${ }^{55}$. The creation of a relationship between doctor and patient focusing on careful maintenance of good oral hygiene is the best way to prevent the appearance of ONJ. Oncologic patients should be well informed about ONJ but also well reassured as to its low absolute risk and also correctly advised regarding the fact that the benefits of cancer therapy far outweigh the potential risk of ONJ.

\section{Conclusion}

We have analyzed the literature on the issue of ONJ due to drug treatment in cases that have been described in recent years. Knowledge of pathogenetic mechanisms and clinical outcomes is essential for the correct care of these patients. We conclude that ONJ must always be carefully investigated and prevented with a multidisciplinary approach involving oncologists, radiation oncologists, and skilled dental practitioners when a cancer patient must begin treatment.

\section{ORCID}

Antonio Fabrizio Nifosì, https://orcid.org/0000-0001-75967767

Mariateresa Zuccarello, https://orcid.org/0000-0001-76660687

Lorenzo Nifosì, https://orcid.org/0000-0002-9175-6975

Vanessa Hervas Saus, https://orcid.org/0000-0002-49178956

Gianfilippo Nifosì, https://orcid.org/0000-0003-4329-6297

\section{Authors' Contributions}

A.F.N. participated in conception and design, data analysis and interpretation, drafting the article, and revising and final approval. M.Z. participated in collection and assembly of data, critical revision, and final approval. L.N. participated in collection and assembly of data, critical revision, and final approval. V.H.S. participated in assembly of data and writing of MRONJ prevention. G.N. participated in conception and design, data analysis and interpretation, drafting the article, and revising and final approval.

\section{Conflict of Interest}

No potential conflict of interest relevant to this article was reported.

\section{References}

1. Kim KM, Rhee Y, Kwon YD, Kwon TG, Lee JK, Kim DY. Medication related osteonecrosis of the jaw: 2015 position statement of the Korean Society for Bone and Mineral Research and the Korean Association of Oral and Maxillofacial Surgeons. J Bone Metab 2015;22:151-65.

2. Adnane L, Trail PA, Taylor I, Wilhelm SM. Sorafenib (BAY 439006, Nexavar), a dual-action inhibitor that targets RAF/MEK/ ERK pathway in tumor cells and tyrosine kinases VEGFR/PDGFR in tumor vasculature. Methods Enzymol 2006;407:597-612.

3. Sun J, Sun Q, Brown MF, Dudgeon C, Chandler J, Xu X, et al. The multi-targeted kinase inhibitor sunitinib induces apoptosis in colon cancer cells via PUMA. PLoS One 2012;7:e43158.

4. Ayllon J, Launay-Vacher V, Medioni J, Cros C, Spano JP, Oudard S. Osteonecrosis of the jaw under bisphosphonate and antiangiogenic therapies: cumulative toxicity profile? Ann Oncol 2009;20:600-1.

5. Beuselinck B, Wolter P, Karadimou A, Elaidi R, Dumez H, Rogiers A, et al. Concomitant oral tyrosine kinase inhibitors and bisphosphonates in advanced renal cell carcinoma with bone metastases. Br J Cancer 2012;107:1665-71.

6. Bozas G, Roy A, Ramasamy V, Maraveyas A. Osteonecrosis of the jaw after a single bisphosphonate infusion in a patient with metastatic renal cancer treated with sunitinib. Onkologie 2010;33:3213.

7. Hoefert S, Eufinger H. Sunitinib may raise the risk of bisphosphonate-related osteonecrosis of the jaw: presentation of three cases. Oral Surg Oral Med Oral Pathol Oral Radiol Endod 2010;110:4639.

8. Agrillo A, Nastro Siniscalchi E, Facchini A, Filiaci F, Ungari C. Osteonecrosis of the jaws in patients assuming bisphosphonates and sunitinib: two case reports. Eur Rev Med Pharmacol Sci 2012;16:952-7.

9. van Cann T, Loyson T, Verbiest A, Clement PM, Bechter O, Willems L, et al. Incidence of medication-related osteonecrosis of the jaw in patients treated with both bone resorption inhibitors and vascular endothelial growth factor receptor tyrosine kinase inhibitors. Support Care Cancer 2018;26:869-78.

10. Koch FP, Walter C, Hansen T, Jäger E, Wagner W. Osteonecrosis of the jaw related to sunitinib. Oral Maxillofac Surg 2011;15:63-6.

11. Fleissig Y, Regev E, Lehman H. Sunitinib related osteonecrosis of jaw: a case report. Oral Surg Oral Med Oral Pathol Oral Radiol 2012;113:e1-3.

12. Garuti F, Camelli V, Spinardi L, Bucci L, Trevisani F. Osteonecrosis of the jaw during sorafenib therapy for hepatocellular carcinoma. Tumori 2016;102(Suppl 2):S69-70.

13. Smidt-Hansen T, Folkmar TB, Fode K, Agerbaek M, Donskov F. Combination of zoledronic acid and targeted therapy is active but may induce osteonecrosis of the jaw in patients with metastatic renal cell carcinoma. J Oral Maxillofac Surg 2013;71:1532-40.

14. Schindler T, Bornmann W, Pellicena P, Miller WT, Clarkson B, Kuriyan J. Structural mechanism for STI-571 inhibition of abelson tyrosine kinase. Science 2000;289:1938-42.

15. Dewar AL, Domaschenz RM, Doherty KV, Hughes TP, Lyons AB. Imatinib inhibits the in vitro development of the monocyte/ macrophage lineage from normal human bone marrow progenitors. Leukemia 2003;17:1713-21.

16. Viviano M, Rossi M, Cocca S. A rare case of osteonecrosis of the jaw related to imatinib. J Korean Assoc Oral Maxillofac Surg 
2017;43:120-4.

17. Abel Mahedi Mohamed H, Nielsen CEN, Schiodt M. Medication related osteonecrosis of the jaws associated with targeted therapy as monotherapy and in combination with antiresorptives. A report of 7 cases from the Copenhagen Cohort. Oral Surg Oral Med Oral Pathol Oral Radiol 2018;125:157-63.

18. Shen G, Ren H, Qiu T, Zhang Z, Zhao W, Yu X, et al. Mammalian target of rapamycin as a therapeutic target in osteoporosis. J Cell Physiol 2018;233:3929-44.

19. Dowling RJ, Topisirovic I, Fonseca BD, Sonenberg N. Dissecting the role of mTOR: lessons from mTOR inhibitors. Biochim Biophys Acta 2010;1804:433-9.

20. Indo Y, Takeshita S, Ishii KA, Hoshii T, Aburatani H, Hirao A, et al. Metabolic regulation of osteoclast differentiation and function. $\mathrm{J}$ Bone Miner Res 2013;28:2392-9.

21. Simone V, Ciavarella S, Brunetti O, Savonarola A, Cives M, Tucci $\mathrm{M}$, et al. Everolimus restrains the paracrine pro-osteoclast activity of breast cancer cells. BMC Cancer 2015; 15:692.

22. Kim DW, Jung YS, Park HS, Jung HD. Osteonecrosis of the jaw related to everolimus: a case report. Br J Oral Maxillofac Surg 2013;51:e302-4.

23. Yamamoto D, Tsubota Y, Utsunomiya T, Sueoka N, Ueda A, Endo $\mathrm{K}$, et al. Osteonecrosis of the jaw associated with everolimus: a case report. Mol Clin Oncol 2017;6:255-7.

24. Giancola F, Campisi G, Lo Russo L, Muzio LL, Di Fede O. Osteonecrosis of the jaw related to everolimus and bisphosphonate: a unique case report? Ann Stomatol (Roma) 2013;4(Suppl 2):20-1.

25. Nifosì AF, Nifosì L, Nifosì G. Osteonecrosis of the jaw in a patient treated with denosumab and temsirolimus. SAJ Case Rep 2017;4:401.

26. Rajabi M, Mousa SA. The role of angiogenesis in cancer treatment. Biomedicines 2017;5:E34.

27. Magremanne M, Lahon M, De Ceulaer J, Reychler H. Unusual bevacizumab-related complication of an oral infection. J Oral Maxillofac Surg 2013;71:53-5.

28. Hartl DM, Bahleda R, Hollebecque A, Bosq J, Massard C, Soria JC. Bevacizumab-induced laryngeal necrosis. Ann Oncol 2012;23:276-8.

29. Mailliez A, Baldini C, Van JT, Servent V, Mallet Y, Bonneterre J. Nasal septum perforation: a side effect of bevacizumab chemotherapy in breast cancer patients. Br J Cancer 2010;103:772-5.

30. Estilo CL, Fornier M, Farooki A, Carlson D, Bohle G 3rd, Huryn JM. Osteonecrosis of the jaw related to bevacizumab. J Clin Oncol 2008;26:4037-8.

31. Greuter S, Schmid F, Ruhstaller T, Thuerlimann B. Bevacizumabassociated osteonecrosis of the jaw. Ann Oncol 2008;19:2091-2.

32. Serra E, Paolantonio M, Spoto G, Mastrangelo F, Tetè S, Dolci M. Bevacizumab-related osteneocrosis of the jaw. Int J Immunopathol Pharmacol 2009;22:1121-3.

33. Wynn RL. Bevacizumab (Avastin): an anti-angiogenic drug associated with osteonecrosis of the jaw. Gen Dent 2011;59:410-3.

34. Dişel U, Beşen AA, Özyılkan Ö, Er E, Canpolat T. A case report of bevacizumab-related osteonecrosis of the jaw: old problem, new culprit. Oral Oncol 2012;48:e2-3.

35. Katsenos S, Christophylakis C, Psathakis K. Osteonecrosis of the jaw in a patient with advanced non-small-cell lung cancer receiving bevacizumab. Arch Bronconeumol 2012;48:187-220.

36. Pakosch D, Papadimas D, Munding J, Kawa D, Kriwalsky MS. Osteonecrosis of the mandible due to anti-angiogenic agent, bevacizumab. Oral Maxillofac Surg 2013;17:303-6.

37. Brunamonti Binello P, Bandelloni R, Labanca M, Buffoli B, Rezzani R, Rodella LF. Osteonecrosis of the jaws and bevacizumab therapy: a case report. Int J Immunopathol Pharmacol 2012;25:78991.

38. Christodoulou C, Pervena A, Klouvas G, Galani E, Falagas ME,
Tsakalos G, et al. Combination of bisphosphonates and antiangiogenic factors induces osteonecrosis of the jaw more frequently than bisphosphonates alone. Oncology 2009;76:209-11.

39. Aragon-Ching JB, Ning YM, Chen CC, Latham L, Guadagnini JP, Gulley JL, et al. Higher incidence of Osteonecrosis of the Jaw (ONJ) in patients with metastatic castration resistant prostate cancer treated with anti-angiogenic agents. Cancer Invest 2009;27:221-6.

40. Guarneri V, Miles D, Robert N, Diéras V, Glaspy J, Smith I, et al. Bevacizumab and osteonecrosis of the jaw: incidence and association with bisphosphonate therapy in three large prospective trials in advanced breast cancer. Breast Cancer Res Treat 2010;122:181-8.

41. McArthur HL, Estilo C, Huryn J, Williams T, Fornier M, Traina TA, et al. Osteonecrosis of the jaw (ONJ) among intravenous (IV) bisphosphonate- and/or bevacizumab-treated patients (pts) at Memorial Sloan-Kettering Cancer Center (MSKCC). J Clin Oncol 2008;26(15 Suppl):9588.

42. Francini F, Pascucci A, Francini E, Miano ST, Bargagli G, Ruggiero $\mathrm{G}$, et al. Osteonecrosis of the jaw in patients with cancer who received zoledronic acid and bevacizumab. J Am Dent Assoc 2011;142:506-13.

43. Hopp RN, Pucci J, Santos-Silva AR, Jorge J. Osteonecrosis after administration of intravitreous bevacizumab. J Oral Maxillofac Surg 2012;70:632-5.

44. Yuan F, Chen Y, Dellian M, Safabakhsh N, Ferrara N, Jain RK. Time-dependent vascular regression and permeability changes in established human tumor xenografts induced by an anti-vascular endothelial growth factor/vascular permeability factor antibody. Proc Natl Acad Sci U S A 1996;93:14765-70.

45. Bettini G, Blandamura S, Saia G, Bedogni A. Bevacizumab-related osteonecrosis of the mandible is a self-limiting disease process. BMJ Case Rep 2012;2012:bcr2012007284.

46. Santos-Silva AR, Belizário Rosa GA, Castro Júnior Gd, Dias RB, Prado Ribeiro AC, Brandão TB. Osteonecrosis of the mandible associated with bevacizumab therapy. Oral Surg Oral Med Oral Pathol Oral Radiol 2013;115:e32-6.

47. Chu QS. Aflibercept (AVE0005): an alternative strategy for inhibiting tumour angiogenesis by vascular endothelial growth factors. Expert Opin Biol Ther 2009;9:263-71.

48. Mawardi H, Enzinger P, McCleary N, Manon R, Villa A, Treister $\mathrm{N}$, et al. Osteonecrosis of the jaw associated with ziv-aflibercept. J Gastrointest Oncol 2016;7:E81-7.

49. Ponzetti A, Pinta F, Spadi R, Mecca C, Fanchini L, Zanini M, et al. Jaw osteonecrosis associated with aflibercept, irinotecan and fluorouracil: attention to oral district. Tumori 2016;102(Suppl 2):S74-7.

50. Langer LF, Clay TM, Morse MA. Update on anti-CTLA-4 antibodies in clinical trials. Expert Opin Biol Ther 2007;7:1245-56.

51. Kong YY, Feige U, Sarosi I, Bolon B, Tafuri A, Morony S, et al. Activated $\mathrm{T}$ cells regulate bone loss and joint destruction in adjuvant arthritis through osteoprotegerin ligand. Nature 1999;402:3049.

52. Owosho AA, Scordo M, Yom SK, Randazzo J, Chapman PB, Huryn JM, et al. Osteonecrosis of the jaw a new complication related to Ipilimumab. Oral Oncol 2015;51:e100-1.

53. Hujoel P, Barasch A, Cunha-Cruz J, Curro FA, Sung AH, Vena $\mathrm{D}$, et al. Osteonecrosis of the jaw and oral hygiene: a case-control study from Condor Dental PBRN. J Dent Hyg 2012;86:32-3.

54. Bonacina R, Mariani U, Villa F, Villa A. Preventive strategies and clinical implications for bisphosphonate-related osteonecrosis of the jaw: a review of 282 patients. J Can Dent Assoc 2011;77:b147.

55. Khan AA, Morrison A, Hanley DA, Felsenberg D, McCauley LK, O'Ryan F, et al.; International Task Force on Osteonecrosis of the Jaw. Diagnosis and management of osteonecrosis of the jaw: a systematic review and international consensus. J Bone Miner Res 2015;30:3-23. 